\title{
Extraction, Characterization, Stability and Biological Activity of Flavonoids Isolated from Chamomile Flowers
}

\author{
Janmejai K Srivastava ${ }^{1,2}$ and Sanjay Gupta ${ }^{1,2,3}$
}

\author{
Department of Urology, ${ }^{1}$ Case Western Reserve University, ${ }^{2}$ University Hospitals Case Medical \\ Center, and ${ }^{3}$ Case Comprehensive Cancer Center, Cleveland, Ohio
}

\begin{abstract}
Dried flowers of Chamomile (Matricaria chamomilla) are largely used for their medicinal properties. In the present study, we examined the pharmacological properties of aqueous and methanolic fraction isolated from two varieties of German chamomile. HPLC-MS analysis of chamomile extract confirmed apigenin-7-Oglucoside as the major constituent of chamomile; some minor glycoside components were observed along with essential oils. These glucosides were highly stable in solution at various temperature ranges and their degradation occured after longterm storage and extraction conditions at different $\mathrm{pH}$ and solvent. Methanolic fraction isolated from chamomile flowers demonstrated higher biologic response in inhibiting cell growth and causing induction of apoptosis in various human cancer cell lines compared to aqueous chamomile fraction. Apigenin glucosides inhibited cancer cell growth through deconjugation of glycosides that occurs in the cellular compartment to produce aglycone, apigenin. Taken together, the pharmacological profile of chamomile extract was dependent upon extraction process, storage conditions which affected the biological activity.
\end{abstract}

Keywords: Chamomile; Apigenin; Apigenin-7-OGlycoside; Apoptosis; Flavonoids

\section{Introduction}

Chamomile has been used for centuries as a medicinal plant mostly for its anti-inflammatory, analgesic, antimicrobial, antispasmodic and sedative properties $(1,2)$. As a member of compositae family, it is widely represented by two known varieties viz.

Received 06/10/09; accepted 07/13/09

Correspondence: Sanjay Gupta, Ph.D., Department of Urology, Case Western Reserve University, University Hospitals Case Medical Center, 10900 Euclid Avenue Cleveland, Ohio 44106, USA. Tel. 216-368-6162, Fax. 216368-0213. email: sanjay.gupta@case.edu
German chamomile (Matricaria chamomilla) and Roman chamomile (Chamaemelum nobile). German chamomile in particular is the most common variety used for medicinal purposes (2). It is known to contain several classes of biologically active compounds including essential oils and several polyphenols. The principal components of the essential oil extracted from chamomile flowers are the terpenoids a-bisabolol and its oxide, azulenes including chamazulene and acetylene derivatives (3). Terpenoids, bisabolol and chamazulene have been shown to possess anti-inflammatory, antiallergic, antispasmodic, antibacterial, antipyretic, ulcerprotective, and antifungal properties (2).

Numerous reports are available on the identification of several phenolic compounds including apigenin, quercetin, and patuletin as glucosides and various acetylated derivatives $(4,5)$. In natural conditions, most of the flavonoids occur as glucosides bound to the sugar moiety and are highly stable and water soluble. In fact, infusion is one of the most popular methods and has been traditionally used as carminative and mild sedative to calm nerves and reduce anxiety, to treat hysteria, nightmares, insomnia and other sleep problems. Additionally, chamomile has been valued as a digestive relaxant and has been used to treat various gastrointestinal disturbances including flatulence, indigestion, diarrhea, anorexia, motion sickness, nausea, and vomiting $(6,7)$. The widespread use and medicinal properties have made chamomile increasingly popular in the form of tea which is consumed at a rate of over one million cups per day. Apart from the existing traditional knowledge on its therapeutic efficacy more work has been conducted in recent years on chamomile to establish its antioxidant, hypocholesteroemic, antiparasitic and anti-aging properties (8-11). More recent studies from our laboratory have evaluated anticancer properties of chamomile (12). The aqueous and methanolic extracts obtained from chamomile exhibited anti-proliferative and apoptotic 
activity in various human cancer cells with minimal effect on normal cells. It has been further demonstrated that apigenin glucosides are the major constituent present in chamomile however studies for their development as anticancer agent needs further investigation. The present study was designed to identify and characterize various glucosides present in chamomile flowers for their stability under different temperatures, $\mathrm{pH}$ and solvent conditions and their affects on the biological activity.

\section{Material and Methods Chemical}

Dry chamomile flowers of Lebanese origin were purchased from Baroody Imports Inc., Main Ave Clifton, New Jersey whereas chamomile flowers of Egyptian origin was purchased from Bec's Tea Nirvana, Cleveland, Ohio. Cell culture medium, RPMI 1640 and DMEM, fetal bovine serum, penicillin-streptomycin cocktail and phosphate buffer saline were purchased from Cellgro Mediatech, Inc. (Herndon, VA). All other reagents were purchased from Sigma (St. Louis, MO) and were of analytical reagent grade or HPLC grade where applicable. Apigenin ( $>98.0 \%$ pure), apigenin 7-O-glucoside ( $>95 \%$ pure), were obtained from Sigma. Tris-EDTA buffer and TBE buffer were procured by Fisher Scientific, (Pittsburgh, PA).

\section{Preparation of extracts}

Dry chamomile flowers were weighed and crushed to powder with a marble pestle and mortar and a $5 \% \mathrm{w} / \mathrm{v}$ suspension was prepared in a flask by adding hot boiled water. The flask was then placed on a shaker (200 rpm) for $4 \mathrm{~h}$ and the temperature was maintained at $37^{\circ} \mathrm{C}$. Essentially similar procedure was used for organic solvent extraction using methanol, ethanol and propanol $(5 \% \mathrm{w} / \mathrm{v})$ and kept on shaker for $4 \mathrm{~h}$ at $200 \mathrm{rpm}$. After shaking, the flask was brought to room temperature the suspension was filtered through a series of Whatman filters and finally passed through 0.22 micron filter (Millipore, Billerica, MA). The filtered aqueous extract was freeze-dried whereas organic extracts were dried at room temperature and stored at $-20^{\circ} \mathrm{C}$ until use. For cell culture studies, the dried material from aqueous extract was weighed and dissolved in culture medium. Organic extracts were dissolved in dimethyl sulfoxide (DMSO) to prepare a stock solution of $100 \mu \mathrm{g} / \mathrm{ml}$ concentration; this was later mixed with the culture media to achieve desired concentration.

\section{Cell lines and culture}

Androgen-refractory human prostate cancer PC3 cells (derived from metastatic prostate cancer in bone) were obtained from American Type Culture Collection (Manassas, VA). Cells were cultured in RPMI 1640 medium with 5\% FBS and 1\% penicillinstreptomycin cocktail at $37^{\circ} \mathrm{C}$ in a humidified atmosphere of $5 \% \mathrm{CO}_{2}$. Other human cancer cell lines used in the study were LNCaP and DU145 (prostate adenocarcinoma), HeLa (cervical adenocarcinoma), T-47D (breast carcinoma), RKO (colon carcinoma) and HT 1080 (fibrosarcoma) cells were cultured in DMEM media supplemented with 10\% FBS and 1\% penicillin-streptomycin cocktail.

\section{Proliferation assay}

The effect of chamomile on the viability of cells was determined by MTT (3-[4, 5-dimethylthiazol-2yl]-2, 5-diphenyl tetrazoliumbromide) assay. Briefly, the cells were plated at $1 \times 10^{4}$ cells per well in 200 $\mu \mathrm{l}$ of complete culture medium containing 25-800 $\mu \mathrm{g} / \mathrm{ml}$ concentrations of freeze-dried aqueous extract in 96-well micro titer plates. A stock solution of methanolic extract was prepared in DMSO and diluted with the culture media to achieve 25-800 $\mu \mathrm{g} / \mathrm{ml}$ final concentrations. The concentration of DMSO remained within maximum permissible concentration of $0.1 \%$ in both control and treated samples. Each concentration of chamomile was repeated in ten wells. After incubation for desired times at $37^{\circ} \mathrm{C}$ in a humidified incubator, cell viability was determined. $50 \mu \mathrm{l}$ MTT $(5 \mathrm{mg} / \mathrm{ml}$ in phosphate buffered saline stock, diluted to working strength $1 \mathrm{mg} / \mathrm{ml}$ with media ) was added to each well and incubated for $2 \mathrm{~h}$ after which the plate was centrifuged at X $600 \mathrm{~g}$ for $5 \mathrm{~min}$ at $4^{\circ} \mathrm{C}$. The MTT solution was removed from the wells by aspiration. After careful removal of the medium, $0.1 \mathrm{ml}$ of buffered DMSO was added to each well, and plates were shaken. The absorbance was recorded on a microplate reader at the wavelength of $540 \mathrm{~nm}$. The effect of chamomile on growth inhibition was assessed as percent cell viability where vehicletreated cells were taken as $100 \%$ viable.

\section{Detection of apoptosis by fluorescence microscopy}

Cells grown in 8 chambered slides (Nunc-Labtek, Nunc, Naperville, IL) were treated with $200 \mu \mathrm{g} / \mathrm{ml}$ aqueous and methanolic chamomile extracts for $24 \mathrm{~h}$. 
Following treatment the cells were washed with PBS and processed for detection apoptosis by using Cytokeratin 18 (CK18) monoclonal antibody, which is an early marker for apoptosis. The fluorescence analysis was performed under the BX51 Olympus microscope by using M 30 Assay Kit (Roche Applied Sciences, Mannheim, Germany) according to vendor's protocol.

\section{HPLC analysis}

All standards, aqueous and organic extracts of chamomile were analyzed on Agilent 1200 HPLC system (Agilent Technologies, Santa Clara, CA) using C-18 column. The mobile phase consists of acetonitrile and water as isocratic solvent $(30: 70 \mathrm{v} / \mathrm{v})$ maintained at a flow rate was $1 \mathrm{ml} / \mathrm{min}$ with injection volume of $5 \mu \mathrm{l}$ and run time of $8 \mathrm{~min}$, respectively. Data were collected at $335 \mathrm{~nm}(\lambda \max$ for the majority of the apigenin glucosides).

\section{Mass spectrometric analysis of apigenin and its glucoside}

Electrospray ionization tandem mass spectrometry was used to identify apigenin and its derivatives in aqueous and methanolic extracts. In brief, chamomile fractions were dissolved in 50\% methanol and introduced onto a Quattro Ultima triple quadruple mass spectrometer (Micromass, Inc., Beverly, MA) at the rate $50 \mu \mathrm{l} / \mathrm{min}$ and analyzed using electrospray ionization both in negative and positive-ion modes. Apigenin and its derivatives were identified using both full and product scans. The capillary and cone voltages were set at $3.5 \mathrm{kV}$ and $50 \mathrm{~V}$ respectively. The desolvation and cone temperatures were set at $250^{\circ} \mathrm{C}$ and $120^{\circ} \mathrm{C}$ respectively. The nitrogen gas flow rate for desolvation and cone was $600 \mathrm{~L} / \mathrm{h}$ and $80 \mathrm{~L} / \mathrm{h}$ respectively. Collision-induced dissociation was obtained using argon gas.

\section{Stability and shelf-life studies with chamomile extracts}

For agent/drug development, information about stability of the product over the period of time and the effect of ambient temperature and other geophysical conditions, the duration which retains the acceptable limit of efficacy and quality is designated as the shelf-life or keeping quality. For such studies, both aqueous and methanolic chamomile extracts were aliquot from the freshly prepared extract and transferred in $1.5 \mathrm{ml}$ eppendorf tubes. These tubes were distributed in 3 groups to store at $-20^{\circ} \mathrm{C}, 4^{\circ} \mathrm{C}$ and at room temperature. The tubes were retracted at $15,30,60$, 90 and 120 days for analysis and dissociation of glucoside bond and were also subjected to efficacy assessment by MTT assay to assess the antiproliferative efficacy in human prostate cancer PC-3 cells. In addition, we performed stability studies on aqueous and methanolic chamomile extracts at different $\mathrm{pH}$, light exposure and long term storage.

Uptake and conversion of apigenin -7-Oglucoside to apigenin by $P C-3$ cells

To access the conversion of chamomile glucosides to aglycone by human prostate cancer cells, $70-80 \%$ confluent PC-3 cells were exposed to $100 \mu \mathrm{g} / \mathrm{mL}$ concentration of methanolic chamomile extract for 12, 24 and $48 \mathrm{~h}$, respectively. After these treatments, media was collected and cells were washed with chilled PBS for 3 times and subjected to preparation of total cell lysate. A portion of culture medium and cell lysate was aliquoted, deproteinized via addition of $0.2 \mathrm{~mL}$ of methanol, vortex-mixed for $60 \mathrm{~s}$, and centrifuged at $2200 \mathrm{~g}$ for $15 \mathrm{~min}$ at $4^{\circ} \mathrm{C}$. The supernatant was collected and subjected to HPLC analysis.

\section{Statistical Analysis}

The values are expressed as mean $\pm \mathrm{SE}$. The significance between the control and treated groups were performed by Student's-' $t$ ' test and $p$ values less than 0.05 were taken as significant in the experiments.

\section{Results and Discussion}

The flower of German chamomile is strongly aromatic and has a bitter taste. We used various extraction solvents per the method described, which yielded pale yellow straw liquid with peculiar fragrance of chamomile. Previous studies have indicated for the presence of various apigenin glucosides in chamomile extract, therefore, we performed HPLC analyses of $99.5 \%$ pure apigenin (MW 270) and apigenin-7-O-glucoside (MW 432) as references, solubilized in methanol at $100 \mathrm{mg} / \mathrm{ml}$ concentration. As shown in Figure 1A, apigenin resolved in a single peak at resolution time $3.0 \mathrm{~min}$ and apigenin-7-O-glucoside at $1.5 \mathrm{~min}$, respectively. Next we used 5\% (v/v) aqueous Lebanese chamomile extract mixed with methanol to obtain HPLC chromatogram. Subsequently, other organic fractions of chamomile extract prepared in methanol, ethanol and propanol were resolved on HPLC. Altogether, a total of 10 peaks were recorded in 
A
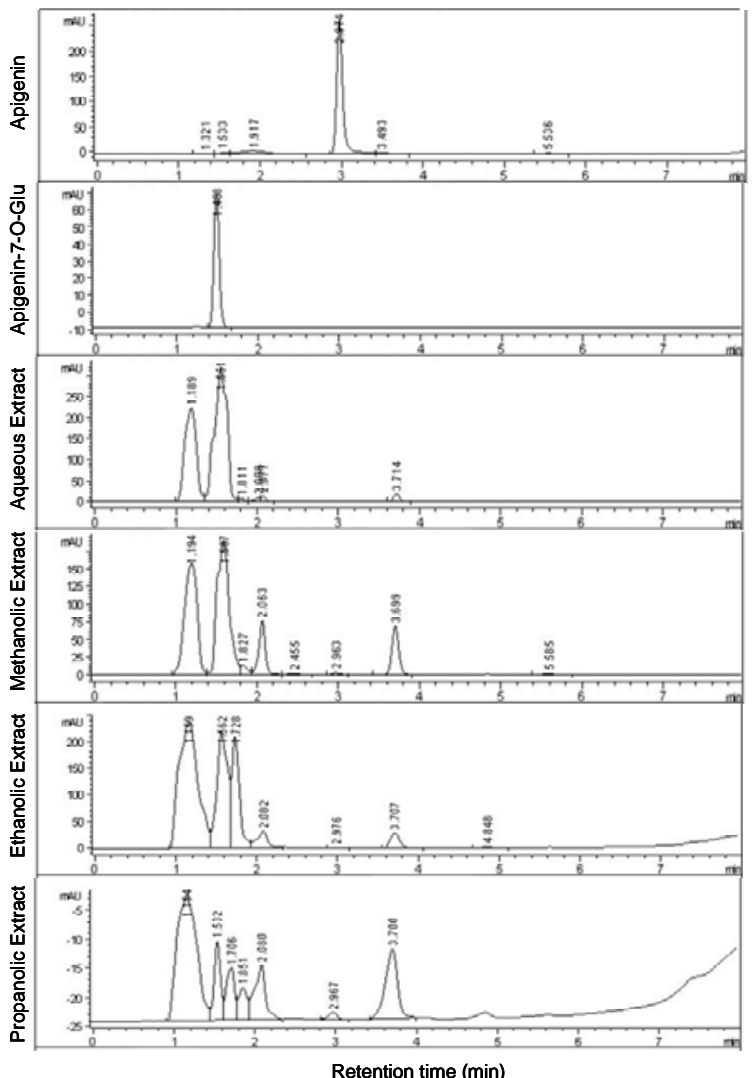

B

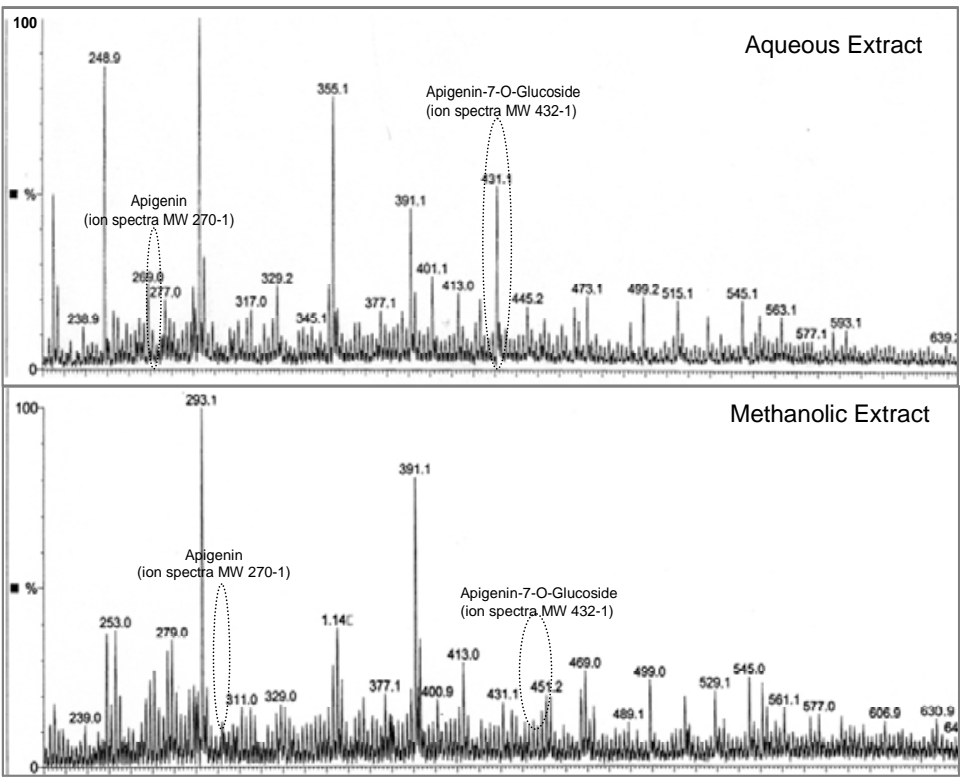

Figure 1. HPLC chromatograms of the $(A)$ aqueous and organic extracts of chamomile demonstrating the presence of various constituents including standards of apigenin and apigenin 7-O-glucoside monitoring relative absorbance at $335 \mathrm{~nm}$ on the same scale. The major constituents observed in aqueous chamomile extracts are apigenin and apigenin-7-O-glucoside. (B) Electrospray product ion mass spectrum of flavonoids extracted from the aqueous and methanolic chamomile extract. This finding confirms the presence of most abundant protonated molecular ion in the extracts for apigenin ( $\mathrm{MW}=270$ ); precursor ion: $\mathrm{m} / \mathrm{z} 271$, and apigenin-7-O-glucoside (MW=432 precursor ion: $\mathrm{m} / \mathrm{z} 433$

aqueous chamomile extract during the scan at wavelength ranging from 200 to $590 \mathrm{~nm}$. In the organic phase scan a total of 21 peaks were noted in the methanolic chamomile extract at the same wavelength (data not shown). Further scanning between 335 to $360 \mathrm{~nm}$ demonstrated apigenin-7-Oglucoside as a major constituent in all the extracts besides having some other minor peaks. To further characterize the presence of constituents in chamomile extracts, we performed LC/MS analysis. Both aqueous and methanolic chamomile extracts demonstrated the presence of mixture of several apigenin glucosides and parent glycone, apigenin which was confirmed by the negative ion spectra obtained by mass spectroscopy (Figure 1B). Previous studies have isolated and identified the presence of both mono- and di-acetylated apigenin-7-glucoside, which are known to undergo rapid ester hydrolysis yielding apigenin-7-O-glucoside. Other apigenin glucosides identified in chamomile extracts were mono-caffeoyl glucoside, mono-acetyl glucoside and mono-malonyl glucoside as well as monoacetyl/mono-malonyl glucosides $(13,14)$. The aqueous chamomile extract have also shown to contain a small fractional (5-7\% of total essential oil) content of the flower (15). This may be due to the relative insolubility of essential oil in aqueous medium. It has been demonstrated that the methanolic chamomile extract have high concentration of apigenin-7-O-glucoside along with several polyphenolic constituents which include caffeic acid, luteolin, luteolin-7-O-glucoside among common flavonoids $(13,14)$. The essential oil content in the methanolic extract consists of chamazulene, $\alpha$-bisabolol, bisabolol oxides A and B, a cyclic ether and different hydrocarbons which are insoluble in 
A Lebanese Chamomile
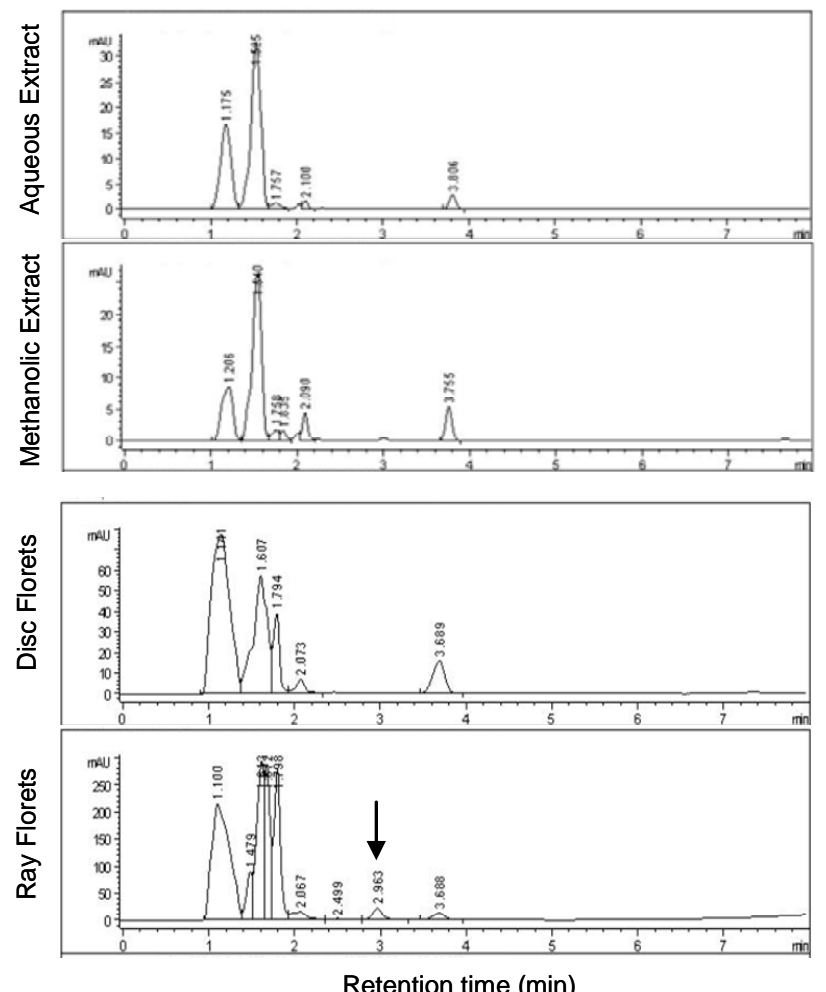

B Egyptian Chamomile
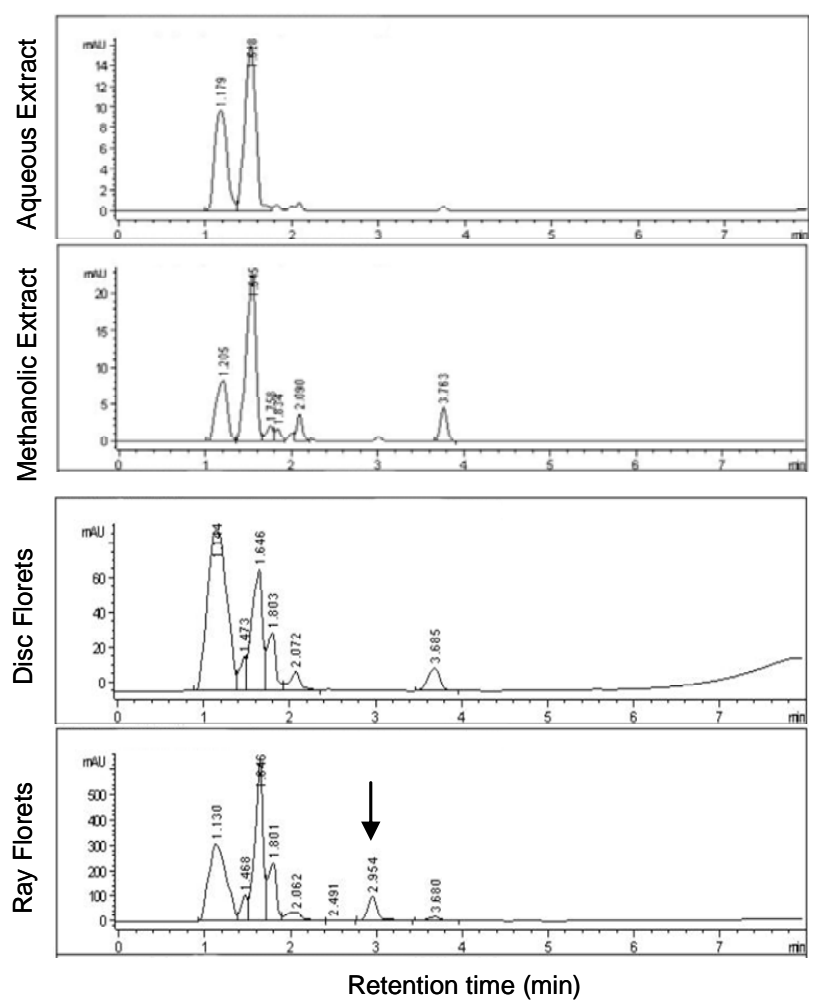

\begin{tabular}{|l|c|c|c|c|}
\hline \multicolumn{1}{|c|}{ Chamomile Flowers } & \multicolumn{2}{|c|}{ Lebanese Chamomile } & \multicolumn{2}{c|}{ Egyptian Chamomile } \\
\hline & Disc Florets & Ray Florets & Disc Florets & Ray Florets \\
\hline Apigenin-7-O-Glucoside (\%) & 38.53 & 39.69 & 36.10 & 39.02 \\
\hline Apigenin (\%) & NIL & 2.33 & NIL & 6.70 \\
\hline
\end{tabular}

Figure 2. Assessment of apigenin 7-O-glucoside and apigenin in the Lebanese and Egyptian varieties of chamomile flowers. The flowers were separated for ray and disc florets, processed for assessment of apigenin-7-O-glucoside and apigenin contents by HPLC analysis. Arrow indicates the peak for apigenin alone.

the aqueous phase $(16,17)$. It is apparent from the extraction that apigenin-7-O-glucoside and various acetylated derivatives are present in chamomile flowers. Glucoside derivatives are readily soluble in water and are extractable whereas loss of glucoside content was observed in the organic phase extraction. Changing of organic solvent from methanol to propanol resulted in lower extraction of apigenin-7-O-glucoside content with an appearance of a peak of other apigenin acetylated derivative at $1.0 \mathrm{~min}$ retention. We also used various grade of methanol ranging from $100 \%$ to $30 \%$ which showed a modest recovery of apigenin-7-O-glucoside in aqueous mixture (data not shown).
Several flavonoids and other phenolic compounds have been identified in various parts of chamomile flowers (18). Studies have shown that apigenin glucosides are mostly concentrated in the ligulate (ray) florets compared to disc florets. Next, we compared the apigenin glucosides in the two varieties of German chamomile, the Lebanese and Egyptian variety, and its presence in the ray and disc florets. Aqueous extracts obtained from both varieties of chamomile showed the abundance of apigenin-7-O-glucoside (data not shown). Methanolic chamomile extract prepared from ray florets of Lebanese and Egyptian chamomile analyzed on HPLC showed the presence of apigenin-7-Oglucoside (39\%) and the aglycone, apigenin; $6.7 \%$ in 
A

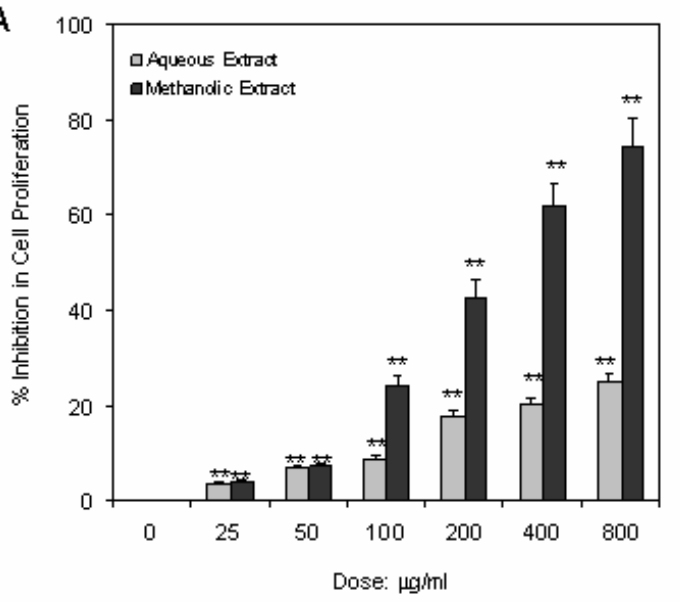

B

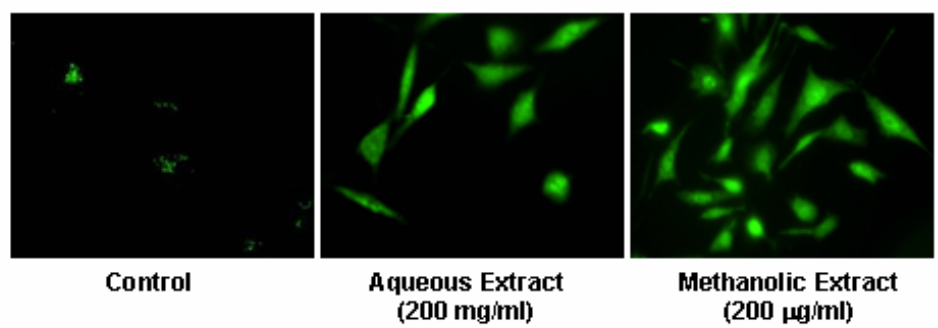

Figure 3. Effect of aqueous and methanolic extracts of chamomile on cell growth inhibition and induction of apoptosis in PC-3 human prostate cancer cells. (A) Cells were exposed to the specified concentrations of aqueous or methanolic chamomile extract for 24 h and viability of the cells was determined by MTT assay. Values represent mean \pm SE of 3 different assays in duplicate **p<0.001 (B) Immunofluorescence detection of apoptosis by M30 CytoDEATH antibody that binds to caspase-cleaved epitope of the cytokeratin 18 cytoskeletal protein, a marker of apoptosis.

the Egyptian and 2.3\% in the Lebanese varieties. Disc florets of chamomile from Lebanese origin showed higher content of apigenin-7-O-glucoside (38.5\%) compared to Egyptian variety (36.1\%); however it did not contain glycone content (Figure 2 $A \& B)$. It is to be noted that the relative quantitative difference in the flavonoid constituents may be attributable to many factors including climatic condition, seasonal changes, geophysical condition and harvest time.

There has been considerable interest in the health promoting effects of flavonoids from plantderived dietary sources including commonly consumed beverages $(1,2)$. Chamomile has a long history of use as tea for various medicinal properties. Studies concerning the major constituent present in chamomile tea reveal apigenin-7-O-glucoside as an important constituent which possesses biological activity. Our earlier studies have demonstrated that apigenin-7-O-glucoside possesses significantly higher anti-proliferative and anticancer activity compared to other glucoside derivatives (12). Numerous studies have evaluated biological effects of individual constituent from plant-derived foods; however limited studies have been done on the effect of extracts obtained from these plants on the biological activity. Therefore, next we evaluated the anti-proliferative and apoptosis inducing effects of aqueous and methanolic chamomile extracts in human prostate cancer PC-3 cells. As shown in Figure 3A, exposure of PC-3 cells to aqueous chamomile extract for $24 \mathrm{~h}$ resulted in dosedependent increase in cell growth inhibition varying from $3-25 \%$ at concentration ranging from 25-800 $\mu \mathrm{g} / \mathrm{ml}$. Similarly, $4-74 \%$ cell growth inhibition was observed after exposure of PC-3 cells to methanolic chamomile extract ranging from 25-800 $\mu \mathrm{g} / \mathrm{ml}$. Exposure of PC-3 cells to both aqueous and methanolic chamomile extract at $200 \mu \mathrm{g} / \mathrm{ml}$ concentration for $24 \mathrm{~h}$ caused induction of apoptosis (Figure 3B). Similar cell growth inhibitory and apoptotic effects were noted in other human cancer cell lines including breast, colon, fibrosarcoma and cervical adenocarcinoma (data not shown). It has been demonstrated that extracts obtained from rosemary, licorice root, soy, green tea and Chinese herb possess biological effects which are in agreement with our results (19-21). Although the observed biological effects with methanolic extract were higher at a given concentration which might be due to additional phenolic compounds noted, both extracts are nevertheless capable of inhibiting cancer cell proliferation and inducing apoptosis.

Next, we performed stability studies on aqueous and methanolic chamomile extracts using different temperature and $\mathrm{pH}$. Light was found to have no significant effect on the degradation of flavonoids both in aqueous and methanolic chamomile extracts. Temperature plays an important role and increasing temperature had an effect on flavonoid degradation. For these studies we considered three temperatures; storage at room temperature $\left(25^{\circ} \mathrm{C}\right)$; refrigerated $4^{\circ} \mathrm{C}$ 


\section{A Aqueous Chamomile Extract}
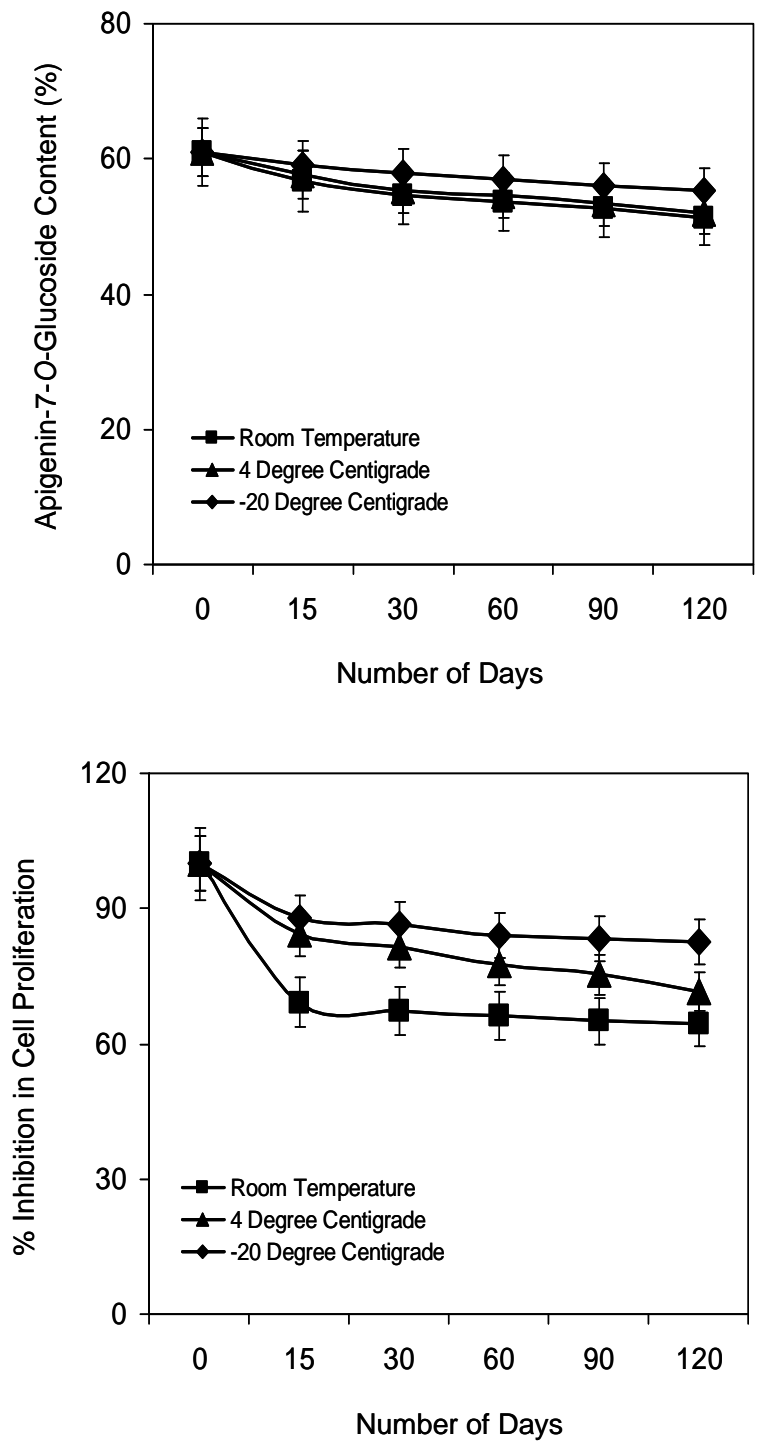

B Methanolic Chamomile Extract
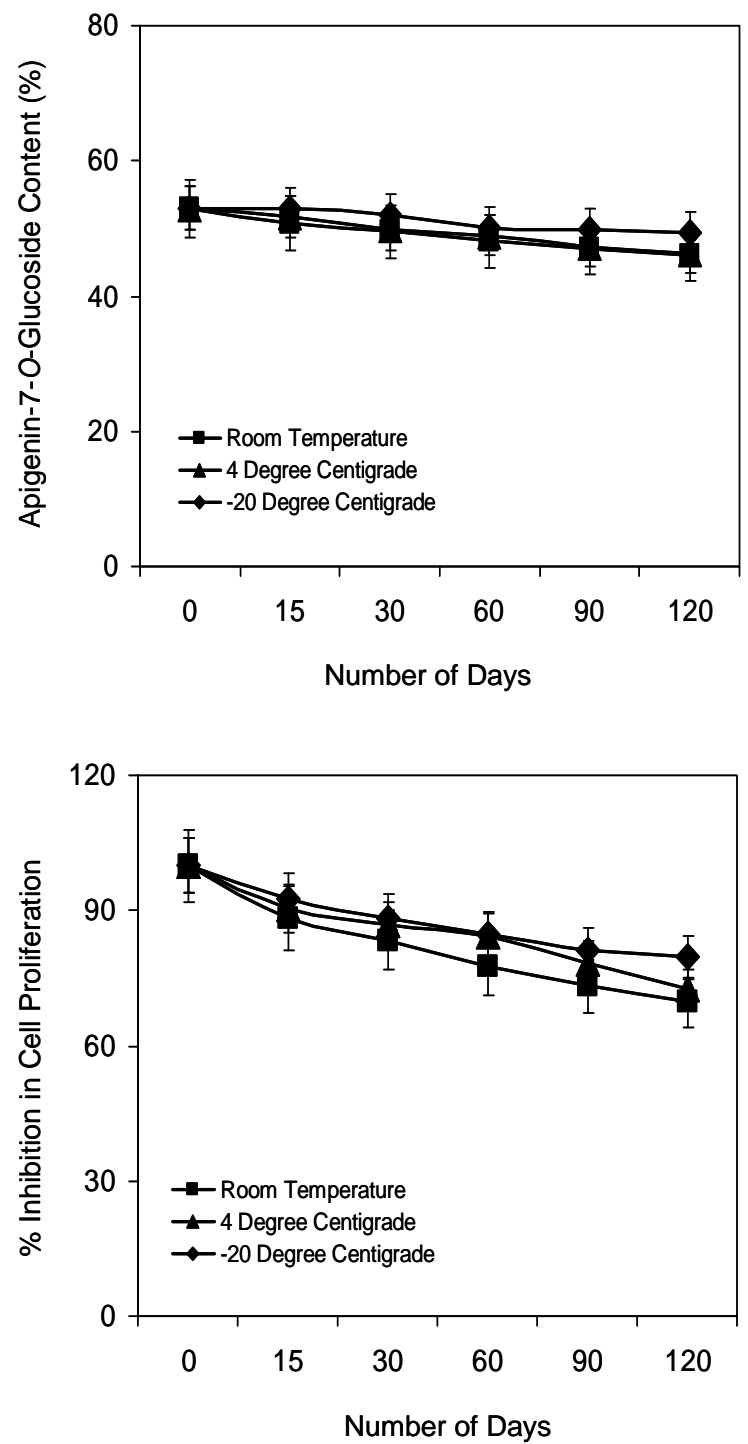

Figure 4. Assessment of product stability and its affect on biological activity of (A) aqueous, (B) methanolic chamomile extract as assessed by apigenin-7-O-glucoside content under various storage conditions by HPLC. The biological activity is determined by using various stored extracts on PC-3 human prostate cancer cells for $24 \mathrm{~h}$ and viability of the cells was determined by MTT assay. Details are described in 'Materials and Methods'.

and frozen at $-20^{\circ} \mathrm{C}$ and followed for 120 days. As shown in Figure $4 \mathrm{~A} \& \mathrm{~B}$, aqueous and methanolic chamomile extracts stored at room temperature and at $4^{0} \mathrm{C}$ demonstrated approximately $10 \%$ degradation of flavonoids up to 120 days. Storage of flavonoids at $-20^{\circ} \mathrm{C}$ caused no significant degradation in the flavonoid content as was ascertained by HPLC profile, which correlated with the biological activity. Further studies have demonstrated noticeable change in the apigenin-7$O$-glucoside content during change in $\mathrm{pH}$. The flavonoids are stable between the $\mathrm{pH} 5$ and 7. The flavonoids tends to degrade with $6 \%$ observed at $\mathrm{pH}$ 8; $9 \%$ at $\mathrm{pH} 9$; and $11-12 \%$ between $\mathrm{pH} 10-12 ; 48 \%$ at $\mathrm{pH} 13$, respectively. No significant degradation of flavonoids was observed at the $\mathrm{pH} 3-5$.

We also assessed the stability of aqueous and methanolic chamomile extract kept at room temperature for 24 months and compared with samples kept at $-20^{\circ} \mathrm{C}$ through HPLC analysis. Long-term storage of aqueous chamomile extract resulted in partial precipitation and increased 
A
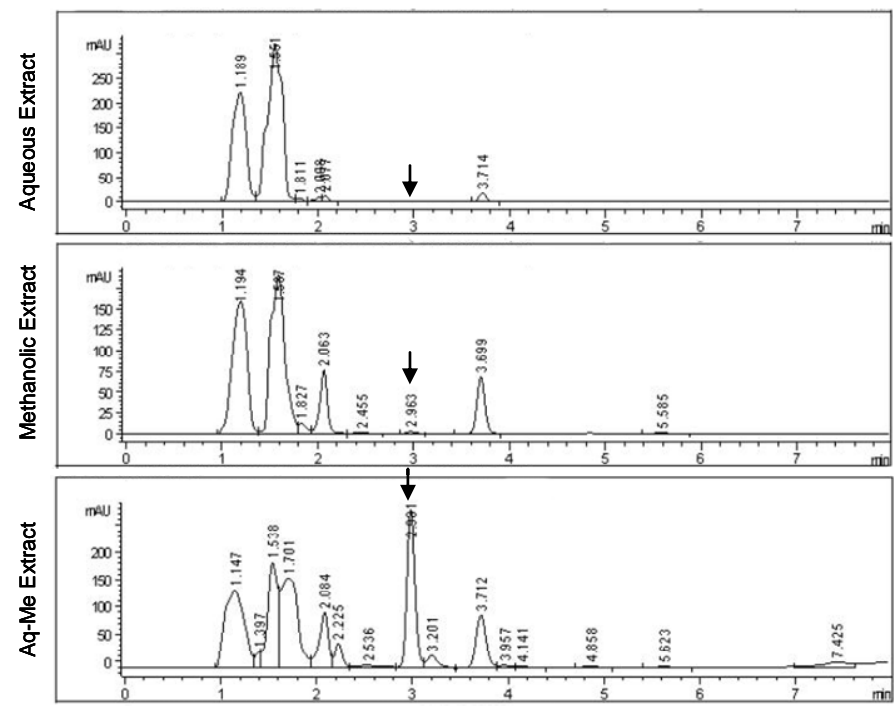

\begin{tabular}{|c|c|c|}
\hline Extract & Apigenin-7-O-Glucoside (\%) & Apigenin (\%) \\
\hline Aqueous Extract & 58.61 & 00 \\
\hline Methanolic Extract & 52.76 & 0.263 \\
\hline Aq-Me Extract & 16.43 & 17.02 \\
\hline
\end{tabular}

B
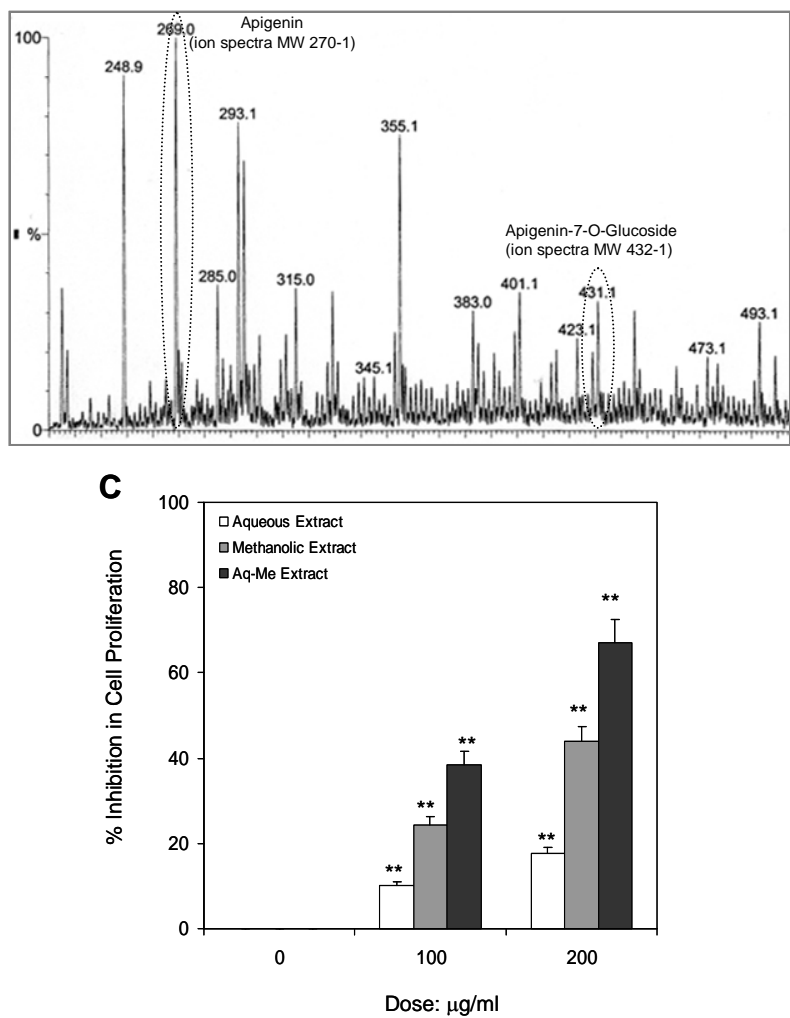

Figure 5. HPLC chromatograms of the $(A)$ aqueous, methanolic and aqueous-methanolic extracts of chamomile demonstrating the presence apigenin 7-O-glucoside and apigenin by monitoring relative absorbance at $335 \mathrm{~nm}$ on the same scale. (B) Electrospray product ion mass spectrum of flavonoids extracted from the aqueous-methanolic chamomile extract. This finding confirms the presence of most abundant protonated molecular ion in the extracts for apigenin ( $\mathrm{MW}=270$ ); precursor ion: m/z 271, and apigenin-7-O-glucoside (MW=432) precursor ion: $\mathrm{m} / \mathrm{z}$ 433. (C) Effect of aqueous, methanolic and aqueous-methanolic extracts of chamomile on cell growth inhibition in PC-3 human prostate cancer cells. The cells were exposed to the specified concentration of chamomile extracts for $24 \mathrm{~h}$ and viability of the cells was determined by MTT assay. Values represent mean \pm SE of 3 different assays in duplicate ${ }^{* \star} p<0.001$

concentration of free aglycone, apigenin in the precipitate. Importantly, the glucoside peak was spliced into several peaks between the retention time of 1.4 to 1.8 minutes and similar trend was noticed in the standard solution of apigenin-7-Oglucoside after long-term storage confirming the conversion of glucoside to aglycone as a common process in solution (data not shown).

In recent years, many bioactive compounds have been identified in food, among them flavonoids isolated from various fruits and vegetables and common beverages have received much attention (21). Epidemiologic and case control studies have shown that these compounds possess various biological and pharmacological properties with beneficial health effects in reducing the risk from several chronic diseases including cancer (22, 23). Previous studies have demonstrated apigenin, as one of the flavonoid which possesses high biological and pharmacological activity (24). Therefore, to further identify the major constituent responsible for the biological activity, we adopted an extraction procedure and ascertained the concentration of apigenin-7-O-glucoside and apigenin. As shown in Figure 5A, the aqueous-methanolic extract showed the presence of high percentage of apigenin (17.0\%) compared to aqueous and methanolic extract along with reduced levels of apigenin-7-O-glucoside (16.4\%). This demonstrates that extraction techniques could significantly alter the glucoside content in the chamomile. These results were confirmed with mass spectroscopy where a reduced peak of apigenin-7-O-glucoside was observed along with emerged peak of apigenin in the same fraction of the chamomile extract (Figure 5B). To confirm whether the biological activity of chamomile is due to the presence of apigenin, human prostate cancer PC-3 cells were exposed to 100 and $200 \mu \mathrm{g} / \mathrm{ml}$ of 
A

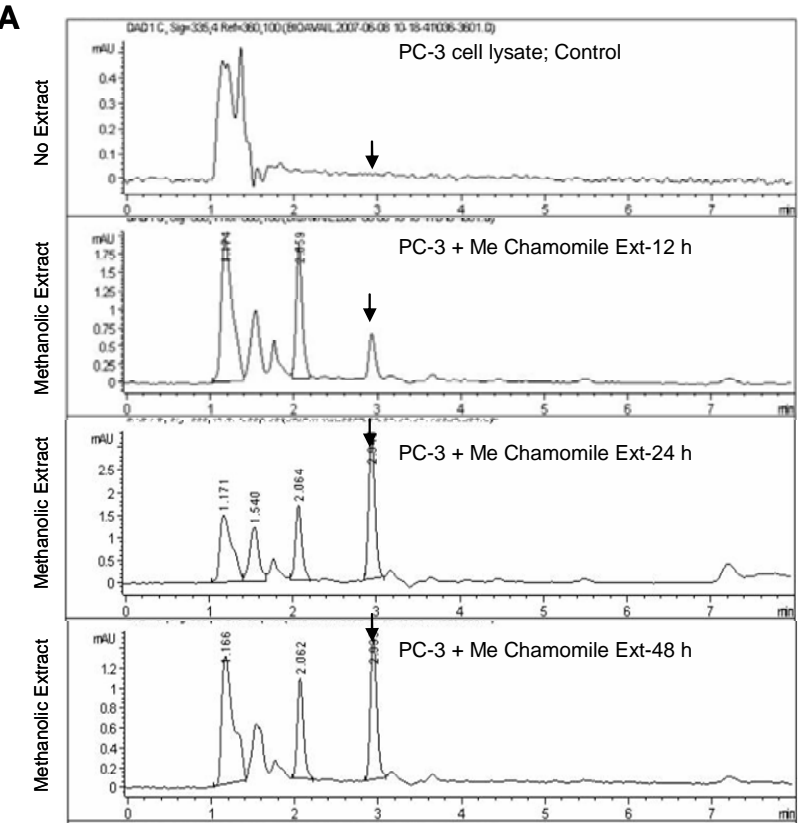

B

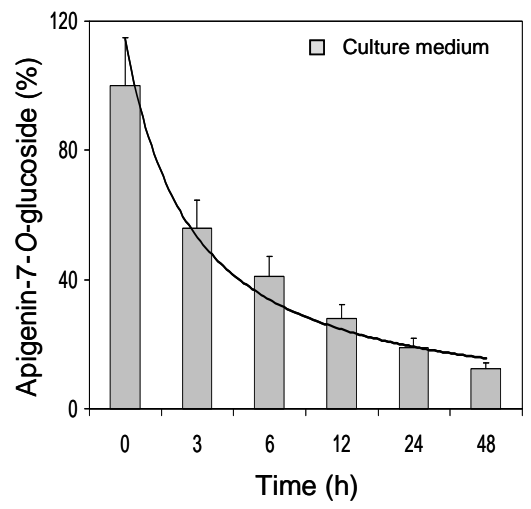

Figure 6. HPLC chromatogram monitoring (A) the relative absorbance at $335 \mathrm{~nm}$ of methanol extract of chamomile after incubation with PC-3 human prostate cancer cells at $37^{\circ} \mathrm{C}$ for 12 and 24 and $48 \mathrm{~h}$ for conversion to aglycone in the cellular compartment (the arrow denotes the aglycone) and (B) the time course decrease in apigenin-7-0-glucoside in the culture medium after incubation with chamomile extract. An exponential time-dependent decrease in apigenin-7-O-glucoside was observed in the culture medium after incubation of PC-3 cells with chamomile extract. Decrease is represented as percent decrease.

aqueous, methanolic and aqueous-methanolic chamomile extracts, respectively. A significant inhibition in proliferation of PC-3 cells was observed with aqueous-methanolic chamomile extracts compared to methanolic and aqueous extract. These results signify apigenin as the major bioactive compound in chamomile extract which possesses anticancer activity.

Next, we evaluated whether human prostate cancer PC-3 cells have capability to convert
apigenin-7-O-glucoside to apigenin. Although studies have shown that aglycone is released by fermentation, autolysis, and deglycosylation via Bglucosidase, B-glucosidase is an enzyme that can effectively deglycosylate various flavonoid glycosides. This enzyme is abundant in the most of the mammalian cells (25). For these studies, PC-3 cells were treated with $100 \mu \mathrm{g} / \mathrm{mL}$ concentration of methanolic chamomile extract for various times interval. As shown in Figure 6A, a representative chromatogram from 12,24 and $48 \mathrm{~h}$ with methanolic chamomile extract incubated with PC-3 cells demonstrated the conversion of apigenin 7-Oglucoside to apigenin probably by the hydrolysis of the 8 -glucoside bond. The increase in apigenin peak observed correlated with the decrease of apigenin-7$O$-glucoside content in the culture medium suggesting (i) apigenin glucosides are present in the cellular compartment, and (ii) cancer cells have potential for deglycosylation of flavonoids and their conversion into aglycone, which is responsible for the anticancer activity (Figure 6B). A previous study has demonstrated that flavonoid glycosides could affect cell proliferation through increased cellular uptake and hydrolysis of the glycosides in human oral squamous carcinoma cells (26).

Flavonoids are polyphenolic compounds that occur ubiquitously in plant-based foods and beverages $(27,28)$. Previous studies have shown that flavonoids content in chamomile may be responsible for its health promoting and anticancer activities where flavonoid glucosides are less effective than their aglycone-the sugar free part of the flavonoid molecule $(12,29)$. Upon absorption the breakdown of flavonoids and release of aglycone occurs in the alimentary canal and later metabolized by methylation or by conjugation with gluconate or sulfate via dual recycling involving both enteric and enterohepatic pathways (30, 31). Our findings demonstrate that apigenin glucosides appear to enter cancer cells and are effectively hydrolyzed within the cells. The concern how various flavonoid bio-products influence the biological activity in vivo could be the subject of further investigation. The combined results from the present study clearly demonstrate that chamomile is an important medicinal plant which contains apigenin possessing high biological activity complexed as apigenin glucoside. Based on these studies apigenin glucosides seem to provide important health benefits to humans. 


\section{Acknowledgement}

This work was supported by grants from United States Public Health Services RO1 AT002709 and RO1 CA108512.

\section{Conflicts of Interest}

No potential conflicts of interest to disclose.

\section{References}

1. McKay DL, Blumberg JB. A review of the bioactivity and potential health benefits of chamomile tea (Matricaria recutita L.). Phytother Res 2006;20:519-30.

2. Gardiner P. Complementary, holistic, and integrative medicine: chamomile. Pediatr Rev 2007;28:16-8.

3. Ganzera M, Schneider P, Stuppner H. Inhibitory effects of the essential oil of chamomile (Matricaria recutita) and its major constituents on human cytochrome P450 enzymes. Life Sci 2006;78:856-61.

4. Svehlikova V, Bennett RN, Mellon FA, et al. Isolation, identification and stability of acylated derivatives of apigenin 7-O-glucoside from chamomile (Chamomilla recutita [L.] Rauschert). Phytochemistry 2004;65:2323-32.

5. Avallone R, Zanoli P, Puia G, Kleinschnitz M, Schreier P, Baraldi M. Pharmacological profile of apigenin, a flavonoid isolated from Matricaria chamomilla. Biochem Pharmacol 2000;59:1387-94.

6. Tyler VE. The honest herbal. $3^{\text {rd }}$ edition, Philadelphia: George F. Stickley Co 1993.

7. Forster HB, Niklas H, Lutz S. Antispasmodic effects of some medicinal plants. Planta Medica 1980;40:309-19.

8. Lee KG, Shibamoto T. Determination of antioxidant potential of volatile extracts isolated from various herbs and spices. J Agric Food Chem 2002;50:4947-52.

9. Babenko NO, Shakhova OH. Age-dependent effects of flavonoids on secretory function of the rat liver. Fiziol $\mathrm{Zh}$ 2005;51:65-9.

10. Di Giorgio C, Delmas F, Tueni M, Cheble E, Khalil T, Balansard G. Alternative and complementary antileishmanial treatments: assessment of the antileishmanial activity of 27 Lebanese plants, including 11 endemic species. J Altern Complement Med 2008;14:157-62.

11. Skovgaard GR, Jensen AS, Sigler ML. Effect of a novel dietary supplement on skin aging in postmenopausal women. Eur J Clin Nutr 2006;60:1201-6.

12. Srivastava JK, Gupta S. Antiproliferative and apoptotic effects of chamomile extract in various human cancer cells. J Agric Food Chem 2007;55:9470-8.

13. Fonseca FN, Tavares MF, Horváth C. Capillary electrochromatography of selected phenolic compounds of Chamomilla recutita. J Chromatogr A 2007;1154:390-9.

14. Redaelli C, Formentini L, Santaniello E. Reversedphase high-performance liquid chromatography analysis of apigenin and its glucosides in flowers of Matricaria chamomilla and chamomile extracts. Planta Med 1981;42:288-92.

15. Heuskin S, Godin B, Leroy P, et al. Fast gas chromatography characterisation of purified semiochemicals from essential oils of Matricaria chamomilla L. (Asteraceae) and Nepeta cataria (Lamiaceae). J Chromatogr A 2009;1216:2768-75.

16. Marczal G, Petri G. Composition of essential oils from native Hungarian camomile. Acta Pharm Hung 1989;59:145-56.

17. Padula LZ, Rondina RV, Coussio JD. Quantitative determination of essential oil, total azulenes and chamazulene in German chamomile (Matricaria chamomilla) cultivated in Argentina. Planta Med 1976;30:273-80.

18. Maier R, Carle R, Kreis W, Reinhard E. Purification and characterization of a flavone 7-O-glucoside-specific glucosidase from ligulate florets of chamomilla recutita. Planta Med 1993;59:436-41.

19. al-Sereiti MR, Abu-Amer KM, Sen P. Pharmacology of rosemary (Rosmarinus officinalis Linn.) and its therapeutic potentials. Indian J Exp Biol 1999;37:124-30.

20. Rossi T, Benassi L, Magnoni C, Ruberto AI, Coppi A, Baggio G. Effects of glycyrrhizin on UVB-irradiated melanoma cells. In Vivo 2005;19:319-22.

21. Kris-Etherton PM, Hecker KD, Bonanome A, Coval SM, Binkoski AE, Hilpert KF, Griel AE, Etherton TD. Bioactive compounds in foods: their role in the prevention of cardiovascular disease and cancer. Am J Med 2002;113 Suppl 9B:71S-88S.

22. Ullah MF, Khan MW. Food as medicine: potential therapeutic tendencies of plant derived polyphenolic compounds. Asian Pac J Cancer Prev 2008;9:187-95.

23. Lee ER, Kang GH, Cho SG. Effect of flavonoids on human health: old subjects but new challenges. Recent Pat Biotechnol 2007;1:139-50.

24. Patel D, Shukla S, Gupta S. Apigenin and cancer chemoprevention: progress, potential and promise (review). Int J Oncol 2007;30:233-45.

25. Day AJ, DuPont MS, Ridley S, Rhodes M, Rhodes MJ, Morgan MR, Williamson G. Deglycosylation of flavonoid and isoflavonoid glycosides by human small intestine and liver beta-glucosidase activity. FEBS Lett 1998;436:71-5.

26. Browning AM, Walle UK, Walle T. Flavonoid glycosides inhibit oral cancer cell proliferation--role of cellular uptake and hydrolysis to the aglycones. J Pharm Pharmacol 2005;57:1037-42.

27. Dashwood RH. Frontiers in polyphenols and cancer prevention. J Nutr 2007;137:267S-269S

28. Soobrattee MA, Bahorun T, Aruoma OI. Chemopreventive actions of polyphenolic compounds in cancer. Biofactors 2006;27:19-35.

29. Chen J, Lin H, Hu M. Metabolism of flavonoids via enteric recycling: role of intestinal disposition. $\mathrm{J}$ Pharmacol Exp Ther 2003;304:1228-35.

30. Hollman PC, de Vries JH, van Leeuwen SD, Mengelers MJ, Katan MB. Absorption of dietary quercetin glycosides and quercetin in healthy ileostomy volunteers. Am J Clin Nutr 1995;62:1276-82.

31. Wang Y, Tang H, Nicholson JK, Hylands PJ, Sampson J, Holmes E. A metabonomic strategy for the detection of the metabolic effects of chamomile (Matricaria recutita L.) ingestion. J Agric Food Chem 2005;53:191-96. 